\title{
COMPOSICIÓN DEL ZOOBENTOS DE LA LAGUNA DE LOBOS, PROVINCIA DE BUENOS AIRES, ARGENTINA.*
}

\author{
A. Rodrigues Capítulo,**A. C. Paggi** e I. César*** \\ * Cont. Cient. N 516 del Instituto de Limnología "Dr. Raúl A. Ringuelet". C.C. 712, 1900 La Plata, Bs. As.. Argentina. \\ ** Investigador CONICET.*** Investigador CIC.
}

Palabras clave: invertebrados bentónicos, densidad, biomasa, lagunas pampásicas, Región Neotropical.

Keywords: benthic invertebrates, density, biomass, pampasic lakes, Neotropical Region.

\section{ABSTRACT \\ ZOOBENTHOS COMPOSITION OF LOBOS POND, PROVINCE OF BUENOS AIRES, ARGENTINE.}

Benthic invertebrates from different horizontal strata samples from Lobos pond, were studied in order to analyse seasonal changes in the composition, numerosity and biomass. Lobos is a polimictic pond (750 Ha; mean depth $1.07 \mathrm{~m}$ ), located in the Pampasic area. The benthic community was dominated by Heleobin parchapii Davis (Hidrobidae). Biomass ranged from 6 to 95 gr. $\mathrm{m}^{-2}$, of which $90 \%$ corresponded to $H$. parchapii. Most frecuent chironomids were Procladius sp. and Coelotanypus lobensis; among microcrustaceans, Acantocyclops robustus (Ciclopoidea), Harpacticoida, Cyprideis salebrosa (Ostracoda), Ilyocriptus sp., Leydigia leidigii, Alonella karua, Bosmina huaronensis and Ceriodaphnia dubia (Cladocera). Diversity ( $\left.\mathrm{H}^{\prime}\right)$ ranged from 0.5 to 2 varying with the different zones of the pond. Distribution of the main taxa throughout the pond and its tributary was discussed.

\section{INTRODUCCION}

Los primeros datos sobre el bentos de las lagunas pampásicas se remontan a 1968-69 donde Ronderos y Orenzanz, analizaron el complejo bentónico y las comunidades perimetrales de cuatro lagunas de la Provincia de Buenos Aires en el marco del Convenio Estudio y Riqueza Ictícola (Salada Grande, San Miguel del Monte, Chascomús y Yalca), las tres últimas ubicadas en proximidad de la laguna de Lobos. Con excepción de análisis taxonómicos sobre varios grupos de especies relacionadas con el complejo bentónico de las lagunas citadas, no ha habido aportes al conocimiento de su ecología.

A partir de 1985 hemos encarado investigaciones limnológicas en la laguna de Lobos, que incluyeron el estudio de la comunidad bentónica (Rodrigues Capítulo et al. en prensa) con especial énfasis en el estudio de las poblaciones de quironómidos (Masaferro et al., 1991, Paggi, 1993). Con este trabajo se completa el inventario faunístico del zoobentos, y se dan a conocer aspectos demográficos relacionados con la distribución espacio-temporal, densidad y biomasa.

\section{ZONA DE ESTUDIO}

La laguna de Lobos (Fig. I) se ubica a $35^{\circ} 17^{\prime} \mathrm{S}$ y a $59^{\circ} 07^{\prime}$ O. al NE de la Prov. de Buenos Aires. Forma parte de la cuenca del río Salado y se desconoce de forma precisa su

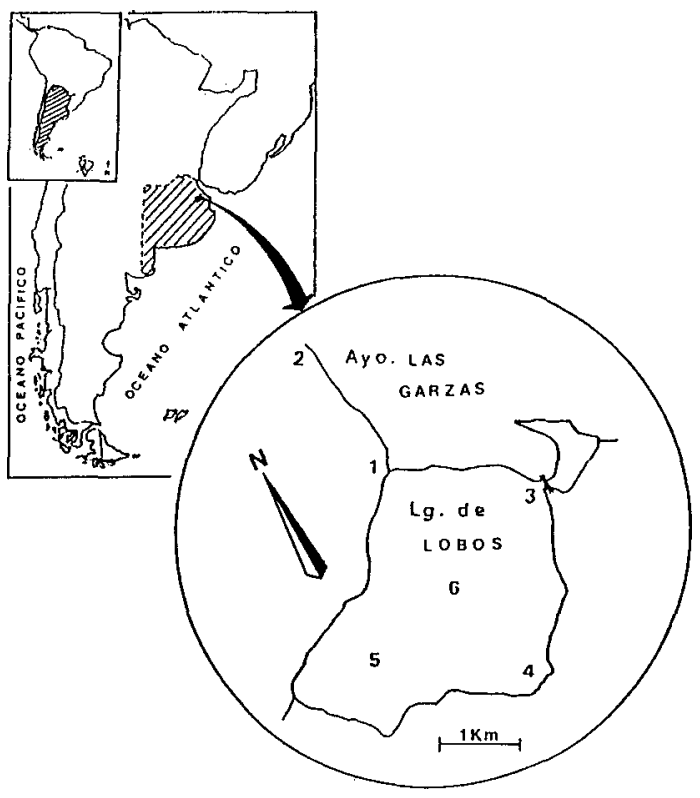

FIGURA 1. Situación geográfica de la Laguna de Lobos y sus estaciones de muestreo. Provincia de Buenos Aires, Argentina.

FIGURE 1. Geographic location of Lobos pond, Provincc of Buenos Aires, Argentina, indicating the sampling sations. 
origen. Su nivel cero $(\mathrm{O})$ se encuentra a 22,5 m.s.n.m. Tiene una superficie de 751,1 Ha y un perímetro de $14,5 \mathrm{~km}$. Su longitud máxima total (LMT) es de $4175 \mathrm{~m}$ (E-W) con un ancho medio de $1800 \mathrm{~m}$ (Sup/ Long. Máx. Tot.); el coeficiente del desarrollo de la línea de costa es de 1,49; la profundidad máxima es de 1,46 $\mathrm{m}$ y la profundidad media es de $1,07 \mathrm{~m}$ (Vol./ Sup.). El volúmen retenido es de 8,06 Hm' (Merlo y Dangavs, 1990).

La precipitación media anual en la zona es de $822 \mathrm{~mm}$ (1970 - 1984) y la temperatura media mensual entre 9 y $23^{\circ} \mathrm{C}$. Se caracteriza por un clima templado húmedo con alternancia en la deficiencia de agua.

Presenta dos afluentes, uno de ellos de caudal permanente, el Ayo. Las Garzas (Ubicado al N), y el otro de caudal temporal que desemboca al SW de la laguna, (Bahía Salvador María). Posee un único efluente (Ayo. Saladillo) al E-NE donde se encuentra el dique regulador del nivel de la laguna.

Otros datos limnológicos, con especial referencia al plancton, se dieron a conocer en el trabajo de Boltovskoy $e t$ al (1990) y Gómez (1990) y sobre materia orgánica en el de Mariñelarena y Ponte Gómez (en prensa).

\section{MATERIAL Y MÉTODOS}

Como resultado de estudios preliminares (Rodrigues Capítulo et al., en prensa) se seleccionaron 6 estaciones de muestreo que correspondieron a diferentes fisonomías de la laguna (Fig. 1); éstas se situaron en el afluente principal (Est. 2, Arroyo Las Garzas), desembocadura del mismo en la laguna (Est. 1, Boca), zona libre de vegetación (Est. 6, Medio), endicamiento del efluente (Est. 3, Vertedero), zonas litorales sin vegetación (Ests. 4 y 5, Palangana y Salvador María respectivamente).

Los muestreos se realizaron con una draga Ekman modificada para trabajar en lugares someros, de $225 \mathrm{~cm}^{2}$ de superficie, que se replicó siempre en 3 ocasiones, durante el período $1 / 1986$ - 10/1986, con una periodicidad trimestral coincidiendo con las estaciones climáticas. Estas se lavaron primariamente "in situ" con el fin de liberar la mayor parte del sedimento fino, con una red de $197 \mathrm{~mm}$ de luz de malla, preservándose el material en formol al $5 \%$.

Submuestreo. En muestras con elevada cantidad de materia orgánica y limoarenosas se procedió a subdividirlas en el laboratorio hasta obtener alícuotas representativas con un fraccionador tipo Folsom según la recomendación de Bonomi (1962).). Se tomó una muestra con draga Ekman de $225 \mathrm{~cm}^{2}$, procediéndose a dividirla en mitades sucesivas con el fraccionador Folsom, hasta alícuotas de $3 \%$ en número de 32. El número de individuos por taxón se dispuso en una matriz básica de datos y a partir de ésta, utilizando el programa de diversidad Estimate H" de Orloci y Kenkel (1985), en base a la ecuación de Shannon y Weaver (1963), se obtuvo la curva acumulada $(\mathrm{H})$, a partir de la cual se estimó el tamaño adecuado de submuestra (Fig.2).

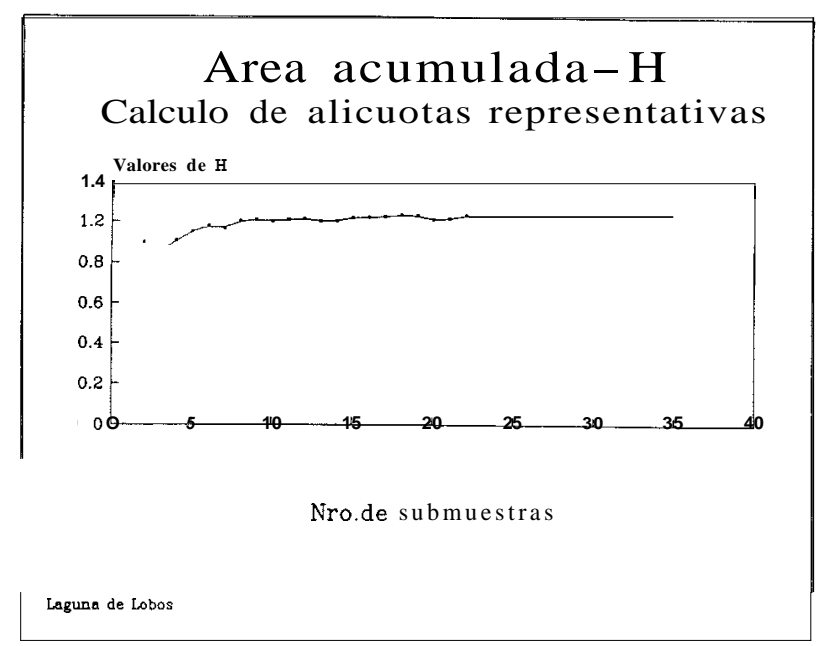

FIGURA 2. Determinación de submuestras del bentos en laboratorio mediante la curva de área acumulada $(\mathrm{H})$

FIGURE 2. Number of benthos subsamples determined in laboratory by using the accumulated area curve $(\mathrm{H})$.

Tratamiento del material en el laboratorio - 1) Obtención de alícuotas representativas de cada draga con un fraccionador Folsom. - 2) Separación de organismos por el método de flotación de Anderson (1959) con solución de sacarosa de densidad $1.12 \mathrm{~g} \cdot \mathrm{ml}^{-1}$. - 3) Tamizado del sedimento con tamices de $250 \mathrm{~mm}$ de luz de malla.- 4) Tinción de los organismos con eritrocina B.- 5) Identificación y recuento primario bajo lupa binocular y con microscopio óptico con contraste de fases (hasta 1000 aumentos) para las determinaciones más finas. -6) La densidad de los organismos se expresó en $\mathrm{n}^{\circ}$ de individuos por $\mathrm{m}^{2}$. Para la estimación del peso individual de los organismos se utilizó una balanza analítica de $0,01 \mathrm{mg}$ de sensibilidad. Los individuos muy pequeños se agruparon en número adecuado, estimando luego el peso promedio. La biomasa del zoobentos (> $250 \mathrm{~mm}$ ) se expresó en gramos de peso húmedo del material fijado con formol, por $\mathrm{m}^{2}$. 
Relación longitud -peso de Heleobia parchapii. Dada la importancia numérica de $\mathrm{H}$. parchapii, se estimó el peso húmedo (W) en relación a la longitud total (Lt) de la concha. Para tal fin se establecieron las ecuaciones de regresión correspondientes a partir del modelo clásico $\mathrm{W}=\mathbf{c} \mathbf{L} \mathbf{t}^{\mathbf{n}}$, donde c y $\mathbf{n}$ se corresponden con las constantes de la ecuación de la recta de regresión. De esta manera se pudo obtener el peso de este molusco a partir de la medición de la longitud total (LT) de la concha, obteniéndose luego la biomasa al multiplicarla por el total de individuos de cada talla.

Diversidad. Para la estimación de la diversidad de los taxones, se tomaron en cuenta el número de individuos y la biomasa por estación y período de muestreo. Fueron calculadas mediante el programa Entropy (Orloci y Kenkel, op.cit.), basado en el índice derivado de la teoría de la información (Shannon y Weaver, 1963).

Análisis de agrupamiento. El estudio de las variaciones espaciales se realizó a través del análisis de agrupamiento por estaciones de muestreo (otu) considerando la presencia y abundancia de los diferentes taxones registrados.

Para la elaboración de las matrices de similitud entre estaciones de muestreo se utilizó el índice de Jaccard, que contempla la presencia y ausencia de las especies y el método de correlación de Pearson, para datos de abundancia. El agrupamiento se realizó mediante el procedimiento "ligamiento promedio no ponderado" UPGMA (Legendre y Legendre, 1983).

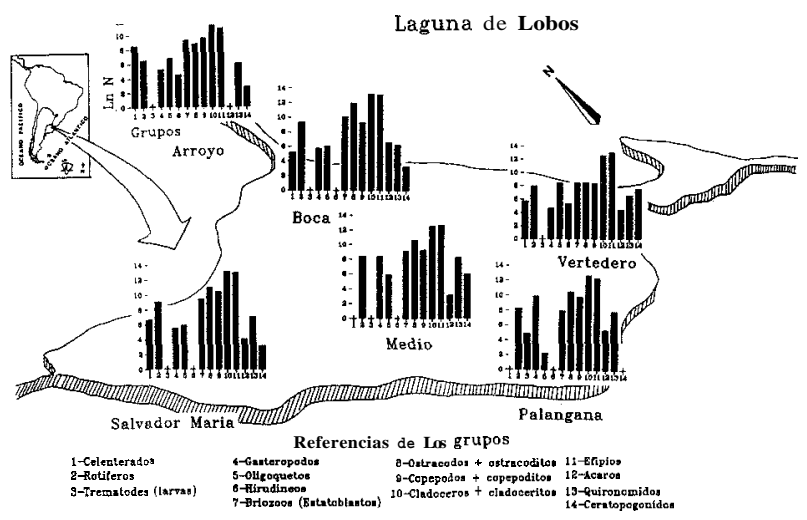

FIGURA 3. Histogramas de numerosidad media estacional en los diferentes áreas de muestreo de la laguna. FIGURE 3. Histograms of mean annual numerosity in the different sapling areas of the pond.

\section{RESULTADOS}

Submuestreo. A partir de los datos de diversidad, estimados mediante el método descrito anteriormente, se observó que la curva alcanzaba la asíntota alrededor de la séptima alícuota acumulada (Fig.2). De esta manera ( $\mathbf{H}$ med.= 1,2727; $\mathrm{H}$ máx.= 2,6391; D.S. de H estimada $=0,001$ ) el $21 \%$ de cada draga fue aceptado como unidad mínima aceptable para cada una de las muestras a analizar.

Distribución y densidad de los invertebrados bentónicos en la laguna. En los histogramas (Fig. 3) se representa la densidad media estacional de los principales taxones examinados, en los ciclogramas de la Fig. 4, su biomasa relativa; la biomasa absoluta se detalla al pie de los ciclogramas (BM). En la Tabla I, la densidad media y desviación standard de los organismos; en la Fig. 5 se grafican las variaciones estacionales de los principales grupos.

El análisis cuantitativo de la fauna reveló un predominio absoluto de Heleobia parchapii Davis, su presencia se registró en forma constante en la mayor parte de la laguna, excepto en estaciones 2 y 3 (afluente principal y desembocadura ), presente aquí sólo al fin del verano. En la estación central (6) y el sector sur (4) se observaron altas densidades durante todo el año con un pico máximo de 41000 ind. $\mathrm{m}^{-2}$ en la primavera.

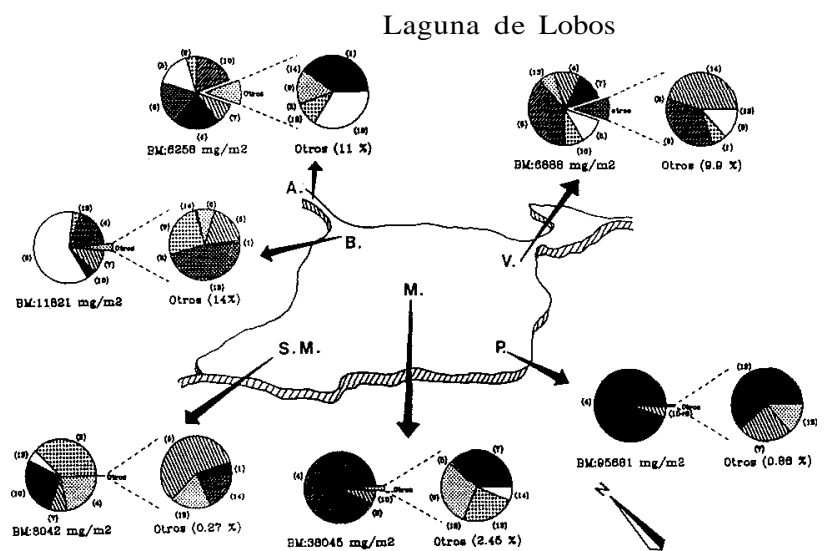

FIGURA 4. Ciclogramas de biomasa relativa y absoluta (BM) de los principales taxones presentes en la laguna.

FIGURE 4. Ciclograms of relative biomass and absolute biomass (BM) of main taxa in the pond. 
Los Hidroida (Hydra sp.) fueron registrados principalmente en primavera y verano en el litoral vegetado; durante el invierno aparecieron solamente en la desembocadura del arroyo Las Garzas donde también se registró en la primavera la mayor densidad numérica (20000 ind. $\mathrm{m}^{-2}$ ), (Tab.I).

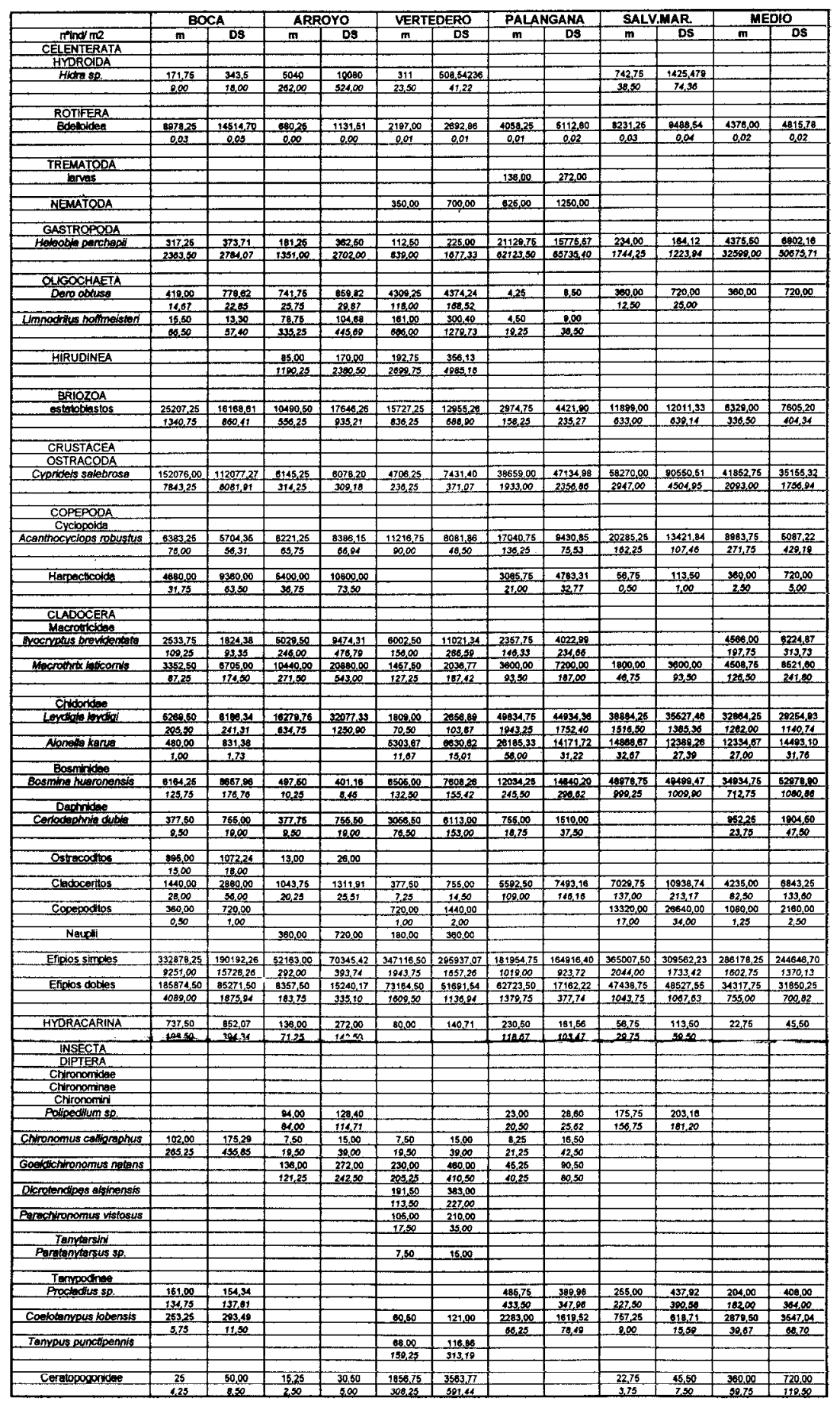

TABLA I: Número promedio de individuos $(\mathbf{m}) \mathrm{m}^{2} \mathrm{y}$ desviación estandar (Ds) de las diferentes estaciones de muestren analizadas. Los valores en italica corresponde a la biomasa expresada en $\mathrm{mg}$. $\mathrm{m}^{-}$.

TABLE I. Mean number of individuals $(\mathrm{m}) \mathrm{m}$ ' and standard deviation (Ds) in the differente analized sampling stations. The values in italic correspnnd tn biomass expressed in mg. $\mathrm{m}$ '. 


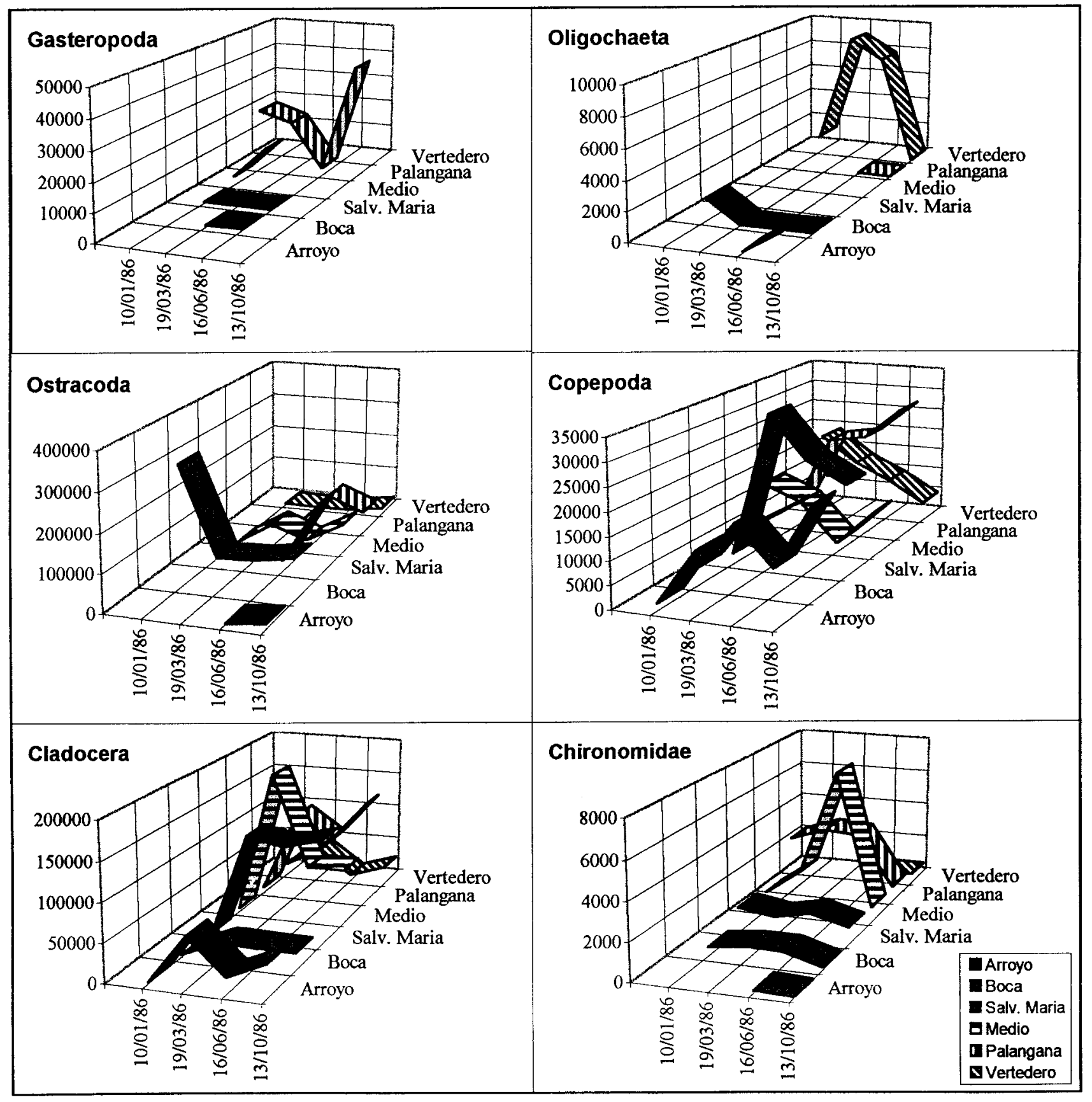

FIGURA 5. Variación espacio - temporal del $\mathrm{N} .^{\circ}$ de ind. $\mathrm{m}$ ' de los principales taxones de invertebrados de la laguna de Lobos FIGURE 5. Spatial and temporal variution of $\mathrm{N}^{\circ}$ of ind. $\mathrm{m}^{-2}$ of rnain invertebratrs taxa in Lobos pond. 
Los rotíferos Bdelloidea se registraron a lo largo de todo el año y prácticamente en todas las estaciones, la mayor densidad numérica correspondió a marzo en estaciones 1 y 5 (litoral).

Los Nematoda predominaron generalmente, en zonas de acumulación de detritos orgánicos (materia vegetal particulada, restos de mudas de cladóceros y de otros artrópodos, etc) y con mayor densidad en primavera y verano. Así, los mayores registros se observaron en la estación 4 (enero) y en la 3 (octubre ) aquí se deposita el material que fluye por el efluente y queda retenido en el dique de nivelación.

Los Oligochaeta Tubificidae (Limnodrilus hoffmeisteri) aparecieron con regularidad a lo largo del año y a densidades relativamente bajas, especialmente en las estaciones 1, 2, 3 y 4; el pico de mayor frecuencia se registró en octubre. Los Naididae (Dero obtusa), aunque casi siempre presentes, manifestaron gran variación espacio temporal. Se halló la máxima densidad, en el dique de nivelación, a fines de la estación cálida, y la mínima en invierno, en el afluente y en la estación 4.

Los Hirudinea aparecieron sólo en el vertedero y en el arroyo Las Garzas, en el verano e invierno.

Hubo 5 especies de Cladocera correspondientes a 4 familias: Ceriodaphnia dubia, Bosmina huaronensis, Leydigia leidigii, Alonella karua, Ilyocryptus brevidentatus y Macrotrix laticornis, las mayores densidades de estos crustáceos se observan durante el final del verano e inicios del otoño. Fue notable la abundancia de efipios de Cladocera en todo el fondo lagunar y durante todo el año. Las mayores concentraciones de efipios simples $(0,3-0,7 \mathrm{~mm})$ se hallaron en las estaciones 3 y 5 en marzo. Los efipios dobles (1-1,5 mm), en su mayoría Daphnidae (C. dubia ?) presentaron su valor máximo en la desembocadura del arroyo en invierno.

Los Copepoda aparecen regularmente a lo largo del año; en enero se registraron Calanoida por única vez en la estación 4. Los Ciclopoida, con Acanthocyclops robustus fueron los más constantes en presencia y numerosidad. Los Harpacticoida estuvieron presentes durante la primavera en las estaciones 1 , 3,4 y 6 y en verano, en las 4 y 5 .

Los Ostracoda, con Cyprideis salebrosa van den Bold presentaron valores de densidad que oscilaron entre $180 \mathrm{y}$ 320000 ind. $\mathrm{m}^{-2}$, correspondiendo ésta última cifra a la desembocadura del arroyo Las Garzas (estación 1).

Los insectos dominantes fueron los Diptera Chironomidae, donde las especies mejor y regularmente representadas entre los Tanipodinae fueron Procladius sp. y Coelotanypus lobensis. La primera de ellas presentó una media de 230 ind. $\mathrm{m}^{-2}$, con un pico más elevado en diciembre y su menor densidad en febrero y junio. Los Chironominae, Chironomus calligraphus y
Dicrotendipes alsinensis fueron frecuentes en la zona litoral vegetada de la laguna, y ocasionales en la zona central.

Los estatoblastos de briozoos fueron registrados casi siempre en gran número y en casi todas las épocas del año, principalmente en los meses de marzo, junio y octubre, con densidades que oscilaron entre 1500 y 38000 ind. $\mathrm{m}^{-2}$. Asimismo se contabilizaron numerosos fragmentos de colonias en enero y octubre, especialmente en el arroyo y su desembocadura y otras zonas litorales asociadas a pequeños afluentes.

Biomasa. La biomasa absoluta promedio hallada para el zoobentos, considerando la fracción superior a las $250 \mathrm{~mm}$, estuvo comprendida entre 6 y 95 gr. $\mathrm{m}^{-2}$ Los valores de biomasa relativa más altos estuvieron siempre relacionados con la presencia de H. parchapii, por ejemplo, en la estación 4 fue del $90 \%$ (Tab. I, Fig. 4), esta especie también dominó durante el verano en las estaciones 1 y 6 . En orden de importancia siguen los Ostracoda (Est. 1 y 5), especialmente en primavera y por último, los Cladocera, con picos máximos en las estaciones 2 y 5 .

Cabe mencionar también que algunos taxa, como los Hirudinea e Hydroida presentaron dominancia ocasional del 57 \% (junio, estación 3) y del $25 \%$ (octubre, estación 2) respectivamente. Por otra parte los oligoquetos, si bien no presentaron dominancias marcadas, tuvieron una presencia anual constante, principalmente en junio y octubre en el afluente principal $(20 \%)$.

Relación longitud- peso de H. parchapii. La ecuación de regresión que mejor se ajustó a los datos observados fue:

$\mathbf{W = 0 . 2 1 6 7}$ LT $^{2.2494}, \quad(\mathrm{~N}=50 ; \mathrm{r}=0.97)$, (Fig.6).

La mayor frecuencia de las tallas se ubicó entre 4,5 y 5,5 $\mathrm{mm}$ de longitud total de la concha, para individuos entre 6 y 9 mgr de peso respectivamente.

Diversidad. La estimación de la diversidad a partir del número de individuos, presentó valores máximos en verano y primavera en la estación 3 (efluente) y a comienzos del invierno en la 1 (arroyo). La diversidad mínima se observó al final del verano en la mayoría de las estaciones. El cálculo de la diversidad considerando la biomasa, tuvo la misma tendencia (Fig. 7).

Análisis de Agrupamiento. La aplicación del ligamiento promedio no ponderado (UPGMA), utilizando la matriz de correlación producto-momento de Pearson, demostró para 


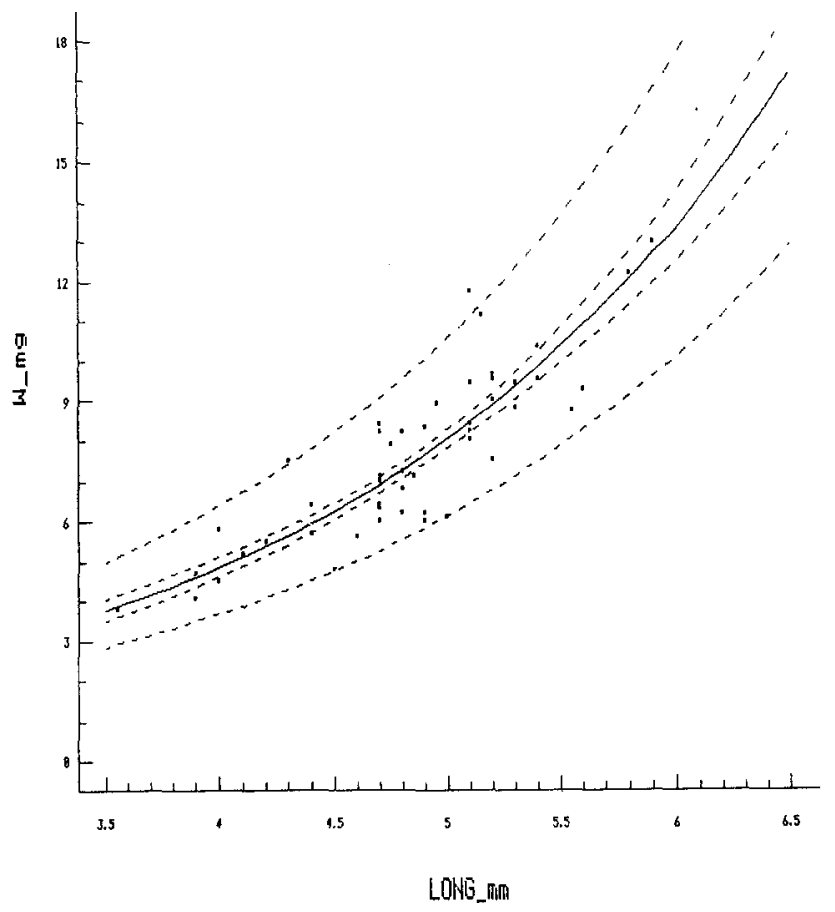

FIGURA 6. Relación entre la longitud total (LT) y el peso formólico (W) de Heleobia parchapii. La línea punteada marca los limites de confianza estadísticos. FIGURE 6. Relationship between total lenght (LT) and formolic weigh (W) of Heleobia parchapii (statistical limits in dotted line).

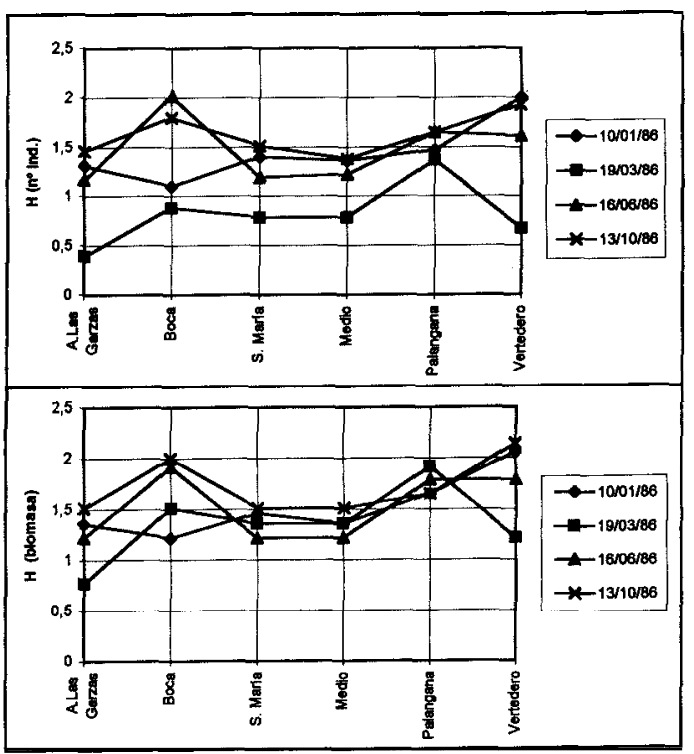

FIGURA 7. índices de diversidad en base al número de individuos y biomasa $(\mathrm{H})$ expresadas en birs de las diferentes estaciones de muestreo en la Laguna de Lobos. FIGURE 7. Numeric diversity index $(\mathrm{H})$ based on number and biomass values expressed in bits in the differente sampling stations. invierno (final del otoño) y primavera la separación de dos grupos evidentes. Uno de ellos formado por la estación Arroyo (2) y el otro por las restantes (1,3,4,5 y 6 ), uniéndose a valores entre 0,2 y 0,5 del dendrograma. Esto demostraría una clara diferenciación del afluente con respecto al resto de la laguna. El segundo grupo mencionado se unió entre sí a valores superiores a 0,9. Para fines del verano (19.3.86) las estaciones Salvador María y Medio $(5,6)$ formaron un grupo que se unió al restante $(1,2,4,3)$ por encima del nivel de 0.8 , (Fig.8).

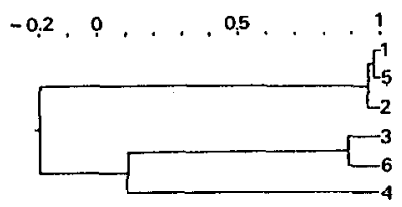

$9-1-86$

$19-3-86$

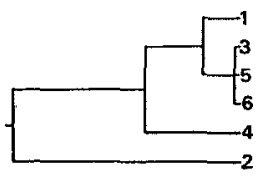

$16-6-86$

$13-10-86$

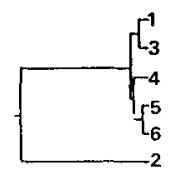

PEARSON

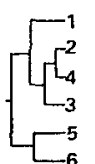

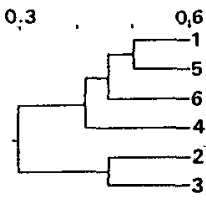

$0, ., 0.5, \ldots, ?$
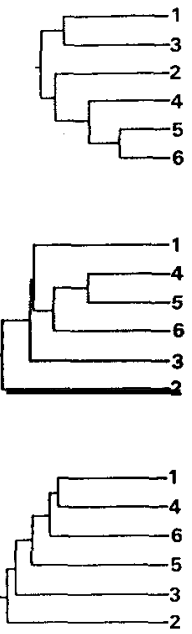

JACCARD
FIGURA 8. Agrupamiento (U.P.G.M.A.) de las estaciones de muestreo según los diferentes taxa a lo largo del año a partir de las matrices de Pearson y Jaccard. FIGURE 7. Cluster analysis (U.P.G.M.A.) of sampling stations according to the different taxa throughtotu the year, bases on Perarson and Jaccard matrices.

Cuando se aplicó UPGMA a las matrices de similitud obtenidas con Jaccard, los agrupamientos fueron similares en líneas generales a los mencionados para Pearson, aunque los niveles de unión de los grupos resultaron inferiores. Sin embargo se observó la formación de un grupo entre las estaciones 2 y 3 , el cual no estuvo reflejado por el método de Pearson. Posiblemente las entradas y salidas de flujo al sistema (estaciones 2 y 3 ) podrían estar determinando al menos en alguna época del año, la presencia de grupos taxonómicos relativamente similares, (Fig. 8). 


\section{DISCUSIÓN}

Una comparación de los organismos del zoobentos de nuestro estudio con los componentes del zooplancton, que fueran estudiados simultáneamente en la laguna de Lobos por Boltovskoy et al (1990) permitió realizar algunas consideraciones. Alonella karua, tal como ocurrió en el plancton, nunca se registró en el arroyo Las Garzas apareciendo solamente en su desembocadura en octubre, esto permitiría confirmar su exclusividad en el área de la laguna. En cambio $L$. leidigii, excepto en enero y octubre (donde sólo se registró en la desembocadura), siempre fueron abundantes en el bentos del arroyo, evidenciando una notable diferencia en relación a su distribución en el plancton. Resulta también interesante la aparición, aunque esporádica, de los harpacticoideos (tipicamente bentónicos) en el plancton, lo que evidencia una escasa distancia y por lo tanto una mezcla entre algunos componentes de ambas comunidades en estos ambientes pampásicos. Por otra parte, a diferencia del plancton, el grupo de los cladóceros en conjunto sicmpre fue relativamente superior al de los copépodos no sólo en el centro de la laguna sino también en los demás sectores estudiados.

Los oligoquetos, tanto naídidos (D. obtusa) como tubifícidos (L. hoffmeisteri), tuvieron una preferencia casi exclusiva por los ambientes de mayor flujo de agua y acúmulo de detritos vegetales, dado que aparecieron fundamentalmente en el arroyo, su desembocadura y en el encauzamiento hacia la salida del efluente.

Los quironómidos C. lobensis y Procladius sp. (siempre presentes en la zona de agua libre de vegetación), nunca se registraron en la estación del arroyo, salvo ocasionalmente en su desembocadura; en cambio Ch. calligraphus, Polypedilum sp. y Goeldichironomus natans se registraron frecuentemente en este afluente. Tanypus punctipennis, de distribución litoral (Masaferro et al., 1991), también aquí fue observada en la zona del efluentc (estación 3) donde hubo acúmulo de detritos.

Dado que son escasos los estudios bentónicos en ambientes lagunares de Argentina, y aunque parezca "caprichosa" la comparación entre limnotopos subtropicales y pampásicos de este tipo, resulta interesante desde el punto de vista ecólogico y zoogeográfico establecer algunas comparaciones de los grupos faunísticos dominantes entre las dos áreas.

En estudios realizados en lagunas subtropicales de la provincia de Corrientes (Bruquetas, 1986; Bonetto et al., 1978), aunque de parámetros morfométricos menores a los de Lobos, se observó en los cladóceros, especies diferentes a las encontradas en la Laguna de Lobos, con la excepción de Alonella karua y Mucrotrix laticornis. Los cladóceros de
Lobos, presentaron en general, densidades inayorcs a lo largo del año que la citadas para las lagunas de Corrientes, en las cuales hubo una marcada reducción de la densidad en el verano y una ausencia de los mismos en zonas menos vegetadas y de mayor profundidad. Los registros de los copépodos de la Laguna de Lobos fueron casi siempre altos, mientras que en los ambientes correntinos fueron escasos, con excepción de los ciclopoideos en la Laguna González.

La fauna de ostrácodos fue citada en las lagunas La Brava y Totoras sin referencia en cuanto a su composición específica y con valores mas bajos a los registrados en Lobos, aunque en esta última, la única especie registrada fue C. salebrosa.

La malacofauna de Lobos estuvo representada casi exclusivainente por H. parchapii; se registraron ocasionalmente ejemplares de Pomacea canaliculata, algunos individuos aislados de Diplodon sp. en el sector Sur litoral de la laguna (Estación 4) y Planorbidae (Pulinonata, Basommatophora) sobre la vegetación costera. La diversidad específica de moluscos de las lagunas Totoras y La Brava siempre fue mayor respecto a Lobos; en el caso de las primeras se citan bivalvos, planórbidos, ampuláridos y ancílidos con una frecuencia regular a lo largo del año principalmente en zonas vegetadas.

Los nematodos presentaron escasa numerosidad en Lobos, en cambio, en las lagunas correntinas este grupo se ha mencionado tanto en el bentos con alto contenido de detritos orgánicos como también en las zonas vegetadas. Este grupo presenta dominancia respecto al resto de las cspecies en la época invernal.

Los oligoquetos de Lobos estuvieron representados en zonas libres de vegetación sólo por dos especies: Limnodrilus hoffmeisteri y Dero obtusa; la primera con densidades regularmente bajas no pasando los 600 individuos $\mathrm{m}$; la segunda presentó densidades variables con un máximo de 9000 individuos $m$ en marzo. En Corrientes los oligoquetos tuvieron una gran variabilidad específica en el litoral lagunar, alcanzando altas densidades en el invierno; se citaron varias especies de Naididae y Enchytraeidae que dominaron el macro y mesobentos local (9700 individuos m ). La densidad de los naídidos en la Laguna Totoras aumentó en el verano (6200 en el litoral y 14000 individuos m ' en el centro de la laguna). En cambio en la Laguna La Brava aumentó la densidad numérica en el invierno (29000 individuos m ), debido posiblemente a la forma de la cubeta, disminuyendo el número de especies y su densidad hacia la primavera y verano. Las especies dominantes fueron Pristina evelinae, $P$. sinclytes y especies de Aulophorus y Dero.

En la laguna de Lobos hubo dos subfainilias de Chironomidae: Chironominae con seis especies y Tanypodinac con tres. Estos 
últimos dominaron fundamentalmente en el agua libre mientras que los primeros en la zona litoral. En las lagunas correntinas mencionadas anteriormente hubo un marcado dominio de los Chironomini sobre los Tanytarsini y dentro de los Tanypodinae, los Pentaneurini. No se mencionan allí los Tanypodini. Dentro de los Chironomini predominaron las especies del género Chironomus con valores fluctuantes anuales entre 6000 y 16000 individuos $m$ en invierno, descendiendo abruptamente estos valores en verano.

La diversidad de especies estuvo relacionada inversamente con la distribución de la acumulación de MO de acuerdo a los valores de Mariñelarena y Ponte Gómez (en prensa) observándose los valores menores de H' en las estaciones 5 y 6 , correspondiendo con un 14 $\%$ de MO, y donde predominó la presencia de H. parchapii. Por otro lado el arroyo Las Garzas presentó valores bajos de diversidad alternativos, probablemente debidos al constante cambio de nivel de agua de acuerdo a las precipitaciones en la zona.

El análisis de agrupamiento de las estaciones teniendo en cuenta los grupos taxonómicos demostró casi siempre el alejamiento de la estación del afluente del resto de las estaciones de la laguna. Por otra parte si bien las estaciones 4,5 y 6 se agrupan en algunas ocasiones, no puede uniformarse ésto para todas las épocas del año. El cambio estacional en la densidad de las diferentes especies estarían definiendo esta situación irregular.

\section{AGRADECIMIENTOS}

Los autores agradecen la valiosa colaboración de las Lic. Andrea Dippolito, Patricia Battistoni y Raquel Sampons, así como a la Dra. María Alejandra Pujals por las determinaciones de cladóceros, copépodos y oligoquetos respectivamente. Asimismo debemos mencionar la colaboración en las tareas de muestreo de los Técnicos Jorge Jauregui y Eduardo Burgueño, así como a las autoridades de la Municipalidad del Partido de Lobos, Provincia de Buenos Aires, por su apoyo logístico.

\section{BIBLIOGRAFIA}

ANDERSON, R.O., 1959. A modified flotation technique for sorting botton fauna samples. Limnol. and Oceanog.4: 223-225. BOLTOVSKOY, A, A. DIPPOLITO, M. FOGGETTA, N. GOMEZ y G. ALVAREZ, 1990. La laguna Lobos y su afluente: Limnologia descriptiva, con especial referencia al plancton. Biología Acuática 14: 37pp.

BONETTO, A.A., M.A. CORRALES, M.E. VARELA, C.A. BONETTO, R.E. VALLEJOS Y Y. ZALOCAR, 1978. Estudios limnológicos en la cuenca del Riachuelo. II. Lagunas Totoras y González. Ecosur 5(9): 17-55.

1978. Estudios limnológicos en la cuenca del Riachuelo. III. Laguna La Brava. Ecosur 5 (9): 57-84.
BONOMI, G., 1962. La dinamica produttiva delle principali popolacioni macrobentonice del Lago di Varese. Mem. Ist. Ital. Idrobiol. 15: 207-254.

BRUQUETAS, I., 1986. Invertebrados que pueblan áreas anegables de la cuenca del Riachuelo (Prov. de Corrientes, Argentina). I: Variaciones temporales en una charca somera vegetada. Rev. Asoc. Cs. Nat. Lit., 17 (2): 217-229.

GÓMEZ, N., 1990. Bacillariophyceae centrales de la Laguna de Lobos, Prov. Buenos Aires, Argentina. Iheringia, ser. Bot. Porto Alegre 40: 65-75.

LEGENDRE, L. y P. LEGENDRE ,1983. Numerical Ecology.- Elsevier Sci. Publ. Co, 419 pp.

MARIÑELARENA, A. y J. PONTE GÓMEZ. Distribución y características de los sedimentos de la laguna de Lobos (Pcia. de Buenos Aires). En prensa en Com. Inv. Cient. Prov. Buernos Aires, Argentina).

MASAFERRO, J., A. C. PAGGI y A. RODRIGUES CAPITUlo, 1991. Estudio poblacional de los quironómidos (Insecta-Diptera) de la Laguna de Lobos, Prov. de Buenos Aires, Argentina. Graellsia. España, 47:129-137.

MERLO, D. y N. V. DANGAVS, 1990. Geología de la laguna de Lobos y su cuenca. Min. As. Agrarios, Prov. Buenos Aires: 1-14.

ORLOCI, L y N.C. KENKEL, 1985. Introduction to data analysis with examples from population and community ecology. International Co-operative Publishing House, USA: $240 \mathrm{pp}$.

PAGGI, A. C.,1993. Formas imaginales y preimaginales de Quironomidos (Diptera) VII. Coelotanypus lobensis sp. nov. y descripción de larva y pupa de C. ringueleti Paggi. 1986 (Chironomidae Tanypodinae). Rev. Soc. Ent. Argentina, 52 (1-4): 5 - 12 .

RODRIGUES CAPITULO, A., A.C. PAGGI, I. CESAR, J. MUZON y J. MASAFERRO. (MS). Estudio preliminar del bentos de la Laguna de Lobos. En Prensa en U.N.L.P.

RODRIGUES CAPITULO, A., A.C. PAGGI, I. CESAR y S. RETTA, (MS). Estudio preliminar del bentos de la laguna de San Miguel del Monte. En Prensa en CIC Prov. Buenos Aires.

RONDEROS, R., J.M. ORENSANZ, 1968-69. El complejo bentónico y las comunidades perimetrales en lagunas de la prov. de Buenos Aires. Resultados preliminares de su estudio: 84-107. En Convenio Estudio Riqueza Ictícola. C.F.I. M.A.A. Prov.Bs.As. 1.

SHANNON, C.E. y W. WEAVER, 1963. The mathematical theory of communication. University of Illinois Press. Urbana.: $177 \mathrm{pp}$. 